Proyecciones Journal of Mathematics

Vol. 33, No 3, pp. 235-243, September 2014.

Universidad Católica del Norte

Antofagasta - Chile

\title{
Statistically pre-Cauchy Fuzzy real-valued sequences defined by Orlicz function
}

\author{
Amar Jyoti Dutta \\ Pragjyotish College, India \\ and \\ Binod Chandra Tripathy \\ Institute of Advanced Study in Science and Technology, India \\ Received: October 2013. Accepted : March 2014
}

\begin{abstract}
In this article we have defined statistically pre-Cauchy sequence of fuzzy real numbers defined by Orlicz function. We have proved a necessary and sufficient condition for a sequence $X=\left(X_{k}\right)$ of fuzzy real numbers to be statistically pre-Cauchy. We have also established some other results.
\end{abstract}

KEY WORDS : Statistically pre-Cauchy; statistically convergence; Orlicz function; fuzzy real numbers.

AMS CLASSIFICATION : 40A05; 46A45; $46 E 30$. 


\section{Introduction}

Fast [8] and Schoenberg [13] independently extended the concept of convergence of a sequence to statistical convergence with the help of natural density function on the set of positive integers. For a set $K \in N$ the density is defined by

$$
\delta(K)=\lim _{n \rightarrow \infty} \frac{1}{n}|\{k \in K: k \leq n\}|,
$$

where $|\{k \in K: k \leq n\}|$ denote the numbers of element in the set.

The notion of statistically pre-Cauchy for real sequences was introduced by Connor, Fridy and Kline [2]. A real-valued sequence $x=\left(x_{k}\right)$ is said to be statistically convergent to a number $L$ if for each $\varepsilon>0, \lim _{n \rightarrow \infty} \frac{1}{n} \mid\{k \leq n$ : $\left.\left|x_{k}-L\right| \geq \varepsilon\right\} \mid=0$, where the vertical bars indicate the number of elements in the enclosed set.

A sequence $x=\left(x_{k}\right)$ is said to be statistically pre-Cauchy if

$$
\lim _{n \rightarrow \infty} \frac{1}{n^{2}}\left|\left\{k \leq n, p \leq n:\left|x_{k}-x_{p}\right| \geq \varepsilon\right\}\right|=0,
$$

for each $\varepsilon \geq 0$.

An Orlicz function is defined by the mapping $M:[0, \infty) \rightarrow[0, \infty)$, which is continuous, non-decreasing and convex with $M(0)=0, M(x)>0$ for $x>0$ and $M(x) \rightarrow \infty$ as $x \rightarrow \infty$. If the convexity of $M$ is replaced by the sub-additivity $M(x+y) \leq M(x)+M(y)$, then it is called the modulus function. An Orlicz function may be bounded or unbounded.

Studies on some important classes of Orlicz sequences from different point of view are found in [4], [7], [10], [14].

A fuzzy real number $X$ is a fuzzy set on $R$, more precisely a mapping $X: R \rightarrow I(=[0,1])$, associating each real number $t$ with its grade of membership $X(t)$, which satisfy the following conditions:

(i) $X$ is normal if there exists $t_{0} \in R$ such that $X\left(t_{0}\right)=1$.

(ii) $X$ upper-semi-continuous if for each $\varepsilon \geq 0, X^{-1}([0, a+))$, is open in the usual topology of $R$, for all $a \in I$. 
(iii) $X$ is convex, if $X(t) \geq X(s) \cap X(r)=\min (X(s), X(r))$, where $s<t<r$.

The class of all upper-semi-continuous, normal, convex fuzzy real numbers is denoted by $R(I)$.

The set of real numbers $R$ can be embedded into $R(I)$, since each $r \in R$ can be regarded as a fuzzy number $\bar{r}$ given by

$$
\bar{r}(t)= \begin{cases}1 & \text { for } t=r \\ 0, & \text { for } t \neq r\end{cases}
$$

The additive identity and multiplicative identity of $R(I)$ are denoted by $\overline{0}$ and $\overline{1}$ respectively.

The $\alpha$-level set of a fuzzy real number $X$ is defined by

$$
[X]^{\alpha}= \begin{cases}\{t \in R: X(t) \geq \alpha\} & \text { for } 0<\alpha \leq 1, \\ \{t \in R: X(t) \geq \alpha\}, & \text { for } \alpha=0 .\end{cases}
$$

Let $D$ be the set of all closed bounded intervals $X=\left[X^{L}, X^{R}\right]$ then we write $X \leq Y$ if and only if $X^{L} \leq Y^{L}$ and $X^{R} \leq Y^{R}$. We can write $d(X, Y)=\max \left[X^{L}-Y^{L}, X^{R}-Y^{R}\right]$, where $X=\left[X^{L}, X^{R}\right]$ and $Y=\left[Y^{L}, Y^{R}\right]$. It is can be easily verify that $(D, d)$ is a complete metric space.

Consider the mapping $\bar{d}: R(I) \times R(I) \rightarrow R$ defined by

$$
\bar{d}(X, Y)=\sup _{0 \leq \alpha \leq 1} d\left(X^{\alpha}, Y^{\alpha}\right), \text { for } X, Y \in R(I) .
$$

Clearly $\bar{d}$ define a metric on $R(I)$.

A fuzzy real-valued sequence $\left(X_{k}\right)$ is a function from the set of natural numbers into $R(I)$. It is denoted by $\left(X_{k}\right)$, where $X_{k} \in R(I)$, for all $k \in N$.

The set $E^{F}$ of sequences taken from $R(I)$ is closed under addition and scalar multiplication defined as follows:

Let $E^{F}$ be the class of sequence of fuzzy real numbers, the linearity of $E^{F}$ can be understand as follows:

For $\left(X_{k}\right),\left(Y_{k}\right) \in E^{F}$ and $r \in R$,

$$
(i)\left(X_{k}\right)+\left(Y_{k}\right)=\left(X_{k}+Y_{k}\right) \in E^{F}
$$




$$
\text { (ii) } r\left(X_{k}\right)=\left(r X_{k}\right) \in E^{F},
$$

where

$$
r\left(X_{k}\right)(t)= \begin{cases}X_{k}\left(r^{-1} t\right), & \text { if } r \neq 0, \\ \overline{0}, & \text { if } r=0 .\end{cases}
$$

A sequence $\left(X_{k}\right)$ of fuzzy real numbers is said to be convergent to the fuzzy real number $X_{0}$, if for every $\varepsilon>0$, there exists $k_{0} \in N$ such that $\bar{d}\left(X_{k}, X_{0}\right)<\varepsilon$, for all $k \geq k_{0}$.

A sequence $\left(X_{k}\right)$ of fuzzy real numbers is said to be bounded if $\sup _{\mathrm{k}} \bar{d}\left(X_{k}, \overline{0}\right)<\infty$, equivalently, if there exist $R^{*}(I)$ such that $\left|X_{k}\right| \leq \mu$ for all $n, k \in N$, where $R^{*}(I)$ denotes the set of all positive fuzzy real numbers.

In recent past different classes of sequences of fuzzy real numbers have been introduced and studied by Altinok, Altin and Et [1], Dutta [3], Savas [12], Mursaleen and Başarir [11], Esi [6], Tripathy and Dutta ([14], [15], [16]), Tripathy and Sarma ([17], [18]) and others.

In this article we have defined statistically pre-Cauchy sequence of fuzzy real numbers and defined by Orlicz function.

A fuzzy real valued sequence $X=\left(X_{k}\right)$ is said to be statistically preCauchy if for each $\varepsilon>0$,

$$
\lim _{n \rightarrow \infty} \frac{1}{n^{2}}\left|\left\{i \leq n, j \leq n: \bar{d}\left(X_{i}, X_{j}\right) \geq \varepsilon\right\}\right|=0 .
$$

\section{Main Results}

Theorem 3.1. Let $M$ be an Orlicz function, then the sequence $\left(X_{k}\right)$ of fuzzy real numbers is statistically pre-Cauchy if and only if

$$
\lim _{n \rightarrow \infty} \frac{1}{n^{2}} \sum_{k, m \leq n} M\left(\frac{\bar{d}\left(X_{k}, X_{m}\right)}{\rho}\right)=0, \text { for some } \rho>0 .
$$

Proof. First we suppose that

$$
\lim _{n \rightarrow \infty} \frac{1}{n^{2}} \sum_{k, m \leq n} M\left(\frac{\bar{d}\left(X_{k}, X_{m}\right)}{\rho}\right)=0, \text { for some } \rho>0,
$$


for some $\rho>0$.

We have

$$
\begin{gathered}
\lim _{n \rightarrow \infty} \frac{1}{n^{2}} \sum_{k, m \leq n} M\left(\frac{\bar{d}\left(X_{k}, X_{m}\right)}{\rho}\right)=\lim _{n \rightarrow \infty} \frac{1}{n^{2}} \sum_{k, m \leq n ; \bar{d}\left(X_{k}, X_{m}\right)<\varepsilon} M\left(\frac{\bar{d}\left(X_{k}, X_{m}\right)}{\rho}\right)+ \\
\lim _{n \rightarrow \infty} \frac{1}{n^{2}} \sum_{k, m \leq n ; \bar{d}\left(X_{k}, X_{m}\right) \geq \varepsilon} M\left(\frac{\bar{d}\left(X_{k}, X_{m}\right)}{\rho}\right) \\
\geq \lim _{n \rightarrow \infty} \frac{1}{n^{2}} \sum_{k, m \leq n ; \bar{d}\left(X_{k}, X_{m}\right) \geq \varepsilon} M\left(\frac{\bar{d}\left(X_{k}, X_{m}\right)}{\rho}\right) \\
\geq M(\varepsilon) \lim _{n \rightarrow \infty} \frac{1}{n^{2}}\left|\left\{(k, m): \bar{d}\left(X_{k}, X_{m}\right)>\varepsilon ; k, m \leq n\right\}\right|
\end{gathered}
$$

Since the L.H.S is zero, we have

$$
\frac{1}{n^{2}}\left|\left\{(k, m): \bar{d}\left(X_{k}, X_{m}\right)>\varepsilon ; k, m \leq n\right\}\right|=0 .
$$

Thus $\left(X_{k}\right)$ is statistically pre-Cauchy.

Conversely suppose that $\left(X_{k}\right)$ is statistically pre-Cauchy. For a given $\varepsilon>0$, choose $\delta>0$ such that $M(\delta)<\frac{\varepsilon}{2}$ and since $M$ is continuous so there exists a positive integer $K$ such that $M(X)<\frac{K}{2}$, for all $X$. We have

$$
\begin{gathered}
\frac{1}{n^{2}} \sum_{k, m \leq n} M\left(\frac{\bar{d}\left(X_{k}, X_{m}\right)}{\rho}\right)=\frac{1}{n^{2}} \sum_{k, m \leq n ; \bar{d}\left(X_{k}, X_{m}\right)<\delta} M\left(\frac{\bar{d}\left(X_{k}, X_{m}\right)}{\rho}\right)+ \\
\frac{1}{n^{2}} \sum_{k, m \leq n ; \bar{d}\left(X_{k}, X_{m}\right) \geq \delta} M\left(\frac{\bar{d}\left(X_{k}, X_{m}\right)}{\rho}\right) \\
\leq M(\delta)+\frac{1}{n^{2}} \sum_{k, m \leq n ; \bar{d}\left(X_{k}, X_{m}\right) \geq \delta} M\left(\frac{\bar{d}\left(X_{k}, X_{m}\right)}{\rho}\right) . \\
\leq \frac{\varepsilon}{2}+\frac{K}{2} \cdot \frac{1}{n^{2}}\left|\left\{(k, m): \bar{d}\left(X_{k}, X_{m}\right)>\varepsilon ; k, m \leq n\right\}\right| .
\end{gathered}
$$

Since $X$ is statistically pre-Cauchy, the R.H.S can be made less than $\varepsilon$. 
Thus we have

$$
\lim _{n \rightarrow \infty} \frac{1}{n^{2}} \sum_{k, m \leq n} M\left(\frac{\bar{d}\left(X_{k}, X_{m}\right)}{\rho}\right)=0 .
$$

This completes the proof.

Theorem 3.2. Let $M$ be an Orlicz function, then the sequence $\left(X_{k}\right)$ of fuzzy real numbers is statistically convergent to $L$ if and only if

$$
\lim _{n \rightarrow \infty} \frac{1}{n} \sum_{k=1}^{n} M\left(\frac{\bar{d}\left(X_{k}, L\right)}{\rho}\right)=0 .
$$

Proof. First we suppose that

$$
\lim _{n \rightarrow \infty} \frac{1}{n} \sum_{k=1}^{n} M\left(\frac{\bar{d}\left(X_{k}, L\right)}{\rho}\right)=0 .
$$

We have

$$
\begin{gathered}
\frac{1}{n} \sum_{k=1}^{n} M\left(\frac{\bar{d}\left(X_{k}, L\right)}{\rho}\right)=\frac{1}{n} \sum_{k=1 ; \bar{d}\left(X_{k}, L\right)<\varepsilon}^{n} M\left(\frac{\bar{d}\left(X_{k}, L\right)}{\rho}\right)+ \\
\frac{1}{n} \sum_{k=1 ; \bar{d}\left(X_{k}, L\right) \geq \varepsilon}^{n} M\left(\frac{\bar{d}\left(X_{k}, L\right)}{\rho}\right) \\
\geq \frac{1}{n} \sum_{k=1 ; \bar{d}\left(X_{k}, L\right) \geq \varepsilon}^{n} M\left(\frac{\bar{d}\left(X_{k}, L\right)}{\rho}\right) \\
\geq M(\varepsilon) \frac{1}{n} \mid\left\{k \leq n: \bar{d}\left(X_{k}, L\right) \geq \varepsilon \mid .\right.
\end{gathered}
$$

Since

$$
\lim _{n \rightarrow \infty} \frac{1}{n} \sum_{k=1}^{n} M\left(\frac{\bar{d}\left(X_{k}, L\right)}{\rho}\right)=0
$$

We have

$$
\lim _{n \rightarrow \infty} \frac{1}{n} \mid\left\{k \leq n: \bar{d}\left(X_{k}, L\right) \geq \varepsilon \mid=0 .\right.
$$

Thus $\left(X_{k}\right)$ is statistically convergent to $L$. 
Converse part can be proved in a similar manner to the previous theorem.

Theorem 3.3. A statistically convergent fuzzy real valued sequence is statistically pre-Cauchy.

Proof. Let the fuzzy real-valued sequence $\left(X_{k}\right)$ is statistically convergent. Let $A \subset N$ be such that $\delta(A)=1$. Select $B \subset A$ such that $A \backslash B$ is finite and $B \times B \subset\left\{(j, k): \bar{d}\left(X_{k}, X_{m}\right)<\varepsilon\right\}$ for some $\varepsilon>0$. Now for $n \in N$, we have

$[\delta(B)]^{2}=\frac{1}{n^{2}} \sum_{k, m \leq m} \chi_{B \times B}(k, m) \leq \frac{1}{n^{2}}\left|\left\{(k, m): \bar{d}\left(X_{k}, X_{m}\right)<\varepsilon ; k, m \leq n\right\}\right|$.

Since

$\lim _{n \rightarrow \infty} \frac{1}{n}[\delta(B)]=1, \Longrightarrow \lim _{n \rightarrow \infty} \frac{1}{n^{2}}\left|\left\{(k, m): \bar{d}\left(X_{k}, X_{m}\right)<\varepsilon ; k, m \leq n\right\}\right|=1$.

This completes the proof.

\section{References}

[1] H. Altinok, Y. Altin, and M. Et: Lacunary almost statistical convergence of fuzzy numbers, Thai J. Math., 2 (2), pp. 265-274, (2004).

[2] J. S. Connor, J. Fridy and J. Kline: Statistically Pre-Cauchy Sequences, Analysis, 14, pp. 311-317, (1994).

[3] A. J. Dutta: Lacunary $p$-absolutely summable sequence of fuzzy real number, Fasc. Math., 46, pp. 58-64, (2011).

[4] A. Esi and M. Et: Some new sequence spaces defined by a sequence of Orliccz functions, Indian j. Pure Appl. Math., 31 (8), pp. 967-972, (2000).

[5] M. Et: On some new Orlicz sequence spaces, J. Analysis, 9, pp. 21-28, (2001).

[6] A. Esi: On some new classes of sequences of fuzzy numbers, Int. J. Math. Anal., 2 (17), pp. 837-844, (2008). 
[7] A. Esi: Generalized Difference Sequence Spaces Defined by Orlicz Functions, Gen. Math., 17 (2), pp. 53-66, (2009).

[8] H. Fast: Sur la Convergence Statistique, Colloq. Math., 2, pp. 241-244, (1951).

[9] J. A. Fridy: On Statistical Convergence, Anal., 5, pp. 301-313, (1985).

[10] V. A. Khan and Q. M. Lohani: Statistically Pre-Cauchy Sequences and Orlicz Functions, Southeast Asian Bull. Math., 31, pp. 1107-1112, (2007).

[11] M. Mursaleen and M. Başarir: On some new sequence spaces of fuzzy numbers, Indian J. Pure Appl. Math., 34 (9), pp. 1351-1357, (2003).

[12] E. Savas: A note on sequence of Fuzzy numbers, Inf. Sc., 124, pp. 297-300, (2000).

[13] I. J. Schoenberg: The integrability of certain functions and related summability methods, Am. Math. Mon., 66, pp. 361-375, (1959).

[14] B. C. Tripathy and A. J. Dutta: On fuzzy real-valued double sequence spaces, Math. Comput. Modelling, 46, pp. (9-10), pp. 1294-1299, (2007).

[15] A. J. Dutta and B. C. Tripathy : On $I$-acceleration convergence of sequences of fuzzy real numbers, Math. Modelling Anal., 17 (4), pp. 549-557, (2012).

[16] B. C. Tripathy and A. J. Dutta: Lacunary bounded variation sequence of fuzzy real numbers, J. Intell. Fuzzy Syst., 24 (1), pp. 185-189, (2013).

[17] B. C. Tripathy and B. Sarma: Sequence spaces of fuzzy real numbers defined by Orlicz functions, Math. Slov. 58 (5), pp. 621-628, (2008).

[18] B. C. Tripathy and B. Sarma: On $I$-convergent double sequences of fuzzy real numbers, Kyungpook Math. J., 52 (2), pp. 189-200, (2012). 


\author{
Amar Jyoti Dutta \\ Department of Mathematics, \\ Pragjyotish College, \\ Guwahati, Assam, \\ India \\ e-mail : amar_iasst@yahoo.co.in \\ and
}

\title{
Binod Chandra Tripathy
}

Mathematical Sciences Division,

Institute of Advanced Study in Science and Technology,

Paschim Baragaon,

Garchuk, Guwahati,

India

e-mail : tripathybc@yahoo.com 